\title{
An efficient approach for shelf space planning and display area allocation in convenience stores
}

\author{
L.K. Chu \\ Department of Industrial and Manufacturing Systems Engineering \\ The University of Hong Kong
}

\begin{abstract}
This paper addresses the problems of shelf space planning and display area allocation in convenience stores. A genetic algorithm is developed to obtain optimum solutions for such problems. A store trial is performed to collect data for analysis and the effects of facing space and display location on product sales are studied. The proposed approach provides a practical and effective means of managing shelf spaces in retail stores.
\end{abstract}

Keywords -Shelf Space Management; Facing Space Elasticity; Display Location Elasticity; Optimisation

\section{INTRODUCTION}

In today's competitive retail industry, retailers are trying to provide better and even unique shopping experiences to their customers. Good shelf space management can attract more sales of some products by increasing their shelf facing space values on the shelves. Studies on visual perception support the view that space has a positive influence on sales for a given product or group of products. If a product is given a large shelf space, it will be in a better position to catch customer's attention and hence a better chance to be sold and more often (Desmet and Renaudin 1998). Also, more shelf facing space can also reduce the possibilities of stock-out. Assigning limited shelf space among many different categories and products is an important problem to address in a retail shop.

Another issue concerns how products are assigned onto different locations in a store. A good knowledge on how display allocations will affect the sales of different products is crucial. While position is found to be far more important than the number of facings on products sales (Dreze, Hoch et al. 1994), the display area allocation among different products within a store is another challenge to retailers.

This study focuses on the various issues of shelf management in convenience stores, which have many specific and important characteristics of their own. A model for obtaining optimal shelf space planning and display area allocation is developed, and an integer encoding genetic algorithm (GA) is employed for obtaining optimum solutions. The approach taken in this study is based on data collected from an established convenience store chain in Hong Kong. This new approach will be appropriate for addressing shelf space planning and display area allocation problems of compact, self-serviced retailing shops with moderate product variety.

\section{II.THE MODEL FOR SHELF SPACE PLANNING AND DISPLAY AREA ALLOCATION}

A flexible approach has been developed for solving the shelf space planning and display area allocation problem. To generate an optimal shelf space and display area allocation plan, the model will only require the data of store sales and information of the constraints of store operations. In order to facilitate the need of different retailers, no limitation on shelf design and product assortment in this model will be imposed. That means, products could be placed on all available locations with appropriate facings, and any product groups (category, sub-category, brand, random product group or one product) could be simultaneously optimised. Also, there is no need for manual adjustment on the trade-off weight between different objectives. In order to minimise manual estimations on parameters, facing space elasticity and display allocation elasticity will be redefined to ensure that both of them can be updated due to the input data. All products or product groups will have their own display allocation elasticities instead of sharing the same value as any other products for taking the same location.

To display a given number of products of different categories to appropriate locations on the shelves of limited space, the model will determine the best ways to utilise the shelf space resource and maximise store's total profit. The following assumptions are made in this model:

1. Demand rate of a product is a function of displayed quantity and displayed allocation of that product.

2. Any possible locations and possible facing quantity for a specific product or product group have the performance record of that product on that allocation before.

3. The unit gross profit of any product is available as an input of the model.

4. The unit width (width per facing) of any product is available as an input of the model.

5. Special requirement on display locations of specific product or product groups are predetermined by store.

6. Grouping of products are predetermined by store.

7. One product or product group can be only placed in one display allocation.

$n$ : Number of products which need to be placed;

$m$ : Number of shelf display locations available to display products;

$g$ : Number of groups the products;

$F$ : Largest number of facing or displayed quantity of one product on the shelves; 
S : An $n \times F$ matrix of facing space elasticity;

L: An $n \times m$ matrix of display area allocation elasticity;

C: An $m \times m$ matrix of distance between every two display allocations;

$\mathbf{R}$ : An $n \times n$ matrix of cross space elasticity between product $i$ and $k$ when placed together with each other;

$d_{i}$ : Average weekly demand for product $i$, $i=1, \ldots, n$;

$p_{i}$ : Unit gross profit of product $i$;

$s_{i x_{i}}$ : Facing space elasticity of $x_{i}$ facings on sales of product $i, \quad s_{i x_{i}}=S\left(i, x_{i}\right)$;

$l_{i y_{i}}$ : Display allocation elasticity of allocation $y_{i}$ on sales of product $i, l_{t y_{i}}=L\left(i, y_{i}\right)$;

$X d_{\iota x_{i}}$ : Average weekly demand of product $i$ when placed with ${ }^{x_{i}}$ facings;

$Y d_{i y_{i}}$ : Average weekly demand of product $i$ when placed on allocation $y_{i}$;

$v_{i x_{i}}$ : Main facing effect of product $i$ when placed with ${ }^{x_{i}}$ facings;

$u_{i y_{i}}$ : Allocation effect of product $i$ when placed on allocation ${ }^{y_{i}}$;

$o_{i}$ : Average facing effect of product $i$;

$h^{i}$ : Average allocation effect of product $i$;

$t_{i}$ : Scale parameter of demand function of product $i$;

$r_{i}$ : Cross effect of product $i$;

$c_{y_{i} y_{k}}$ : Minimal Euclidean distance between allocation $y_{i}$ and $y_{k}, \quad c_{y_{i} y_{k}}=C\left(y_{i}, y_{k}\right)$, $c_{y_{i} y_{k}} \geq 0$;

$w_{i}$ : Unit width per facing of product $i$;

$A_{j}$ : Total width of display allocation $j$, $j=1, \ldots, m$

$\gamma_{i k}$ : Cross space elasticity between product $i$ and $k$, when placed together, $\gamma_{i k}=R(i, k)$; $\theta_{i k}$ : Distance effect parameter on allocation elasticity, $\theta_{i k} \leq 0$

Decision variables:

$x_{i}$ : Total number of facing product $i$ displayed on shelves;

$y_{i}$ : Display allocation of product $i$;

$z_{q}$ : Display allocation of product group $q$, $q=1, \ldots, g$

$b_{i j}$ : The $(0,1)$ decision variable of the allocation on which product $i$ placed. If $y_{i}=j, b_{i j}=1$; and if $y_{i} \neq j, \quad b_{i j}=0$;

$u_{i q}$ : The $(0,1)$ decision variable of whether product $i$ belongs to group $q$.

By assuming that the demand of product is a function of displayed quantity, displayed allocation and cross effect, the demand functions are formulated as follows: $d_{i}=o_{i} h_{i} t_{i} r_{i} ; X d_{i x_{i}}=v_{i x_{i}} h_{i} t_{i} r_{i} ; Y d_{i y_{i}}=u_{i y_{i}} o_{i} t_{i} r_{i}$ where $h_{i}=\bar{u}_{i y_{i}}$ and $y_{i} \leq m ; o_{i}=\bar{v}_{i x_{i}}$ and $x_{i} \leq F$.

Thus, the space elasticity is $s_{i x_{i}}=\frac{X d_{i x_{i}}}{d_{i}}=\frac{v_{i x_{i}}}{\bar{v}_{i x_{i}}}$ and the allocation elasticity $l_{i y_{i}}=\frac{Y d_{i y_{i}}}{d_{i}}=\frac{u_{i y_{i}}}{\bar{u}_{i y_{i}}} . \quad$ The demand function of product $i$ can be given as $D_{i}=d_{i} s_{i x_{i}} l_{y_{i}} \prod_{k \neq i}^{n}\left\lfloor x_{k}^{\gamma_{i k}} c_{y_{i} y_{k}}^{\theta_{i k}}\right\rfloor$. The total gross profit of whole store can be given as $T P=\sum_{i=1}^{n} p_{i} D_{i}=\sum_{i=1}^{n}\left[p_{i} d_{i} s_{i x_{i}} l_{i y_{i}} \prod_{k \neq i}^{n}\left(x_{k}^{\gamma_{i k}} c_{y_{i} y_{k}}^{\theta_{i k}}\right)\right] \quad$ subject to $\quad x_{i} \leq F \quad ; \quad y_{i} \leq m \quad ; \quad \sum_{i=1}^{n} w_{i} x_{i} b_{i j} \leq A_{j} \quad$ for $j=1,2, \ldots, m ; \quad d_{i} \geq 0 ; \quad c_{y_{i} y_{k}} \geq 0 ; \quad b_{i j} \in\{0,1\}$.

For ease of use of the model, the facing space elasticity is redefined as the ratio between the average weekly demand of product $i$ when placed with $x_{i}$ facings to the general average weekly demand $d_{i}$.

The display allocation elasticity has also been redefined as the ratio between average weekly demand of product $i$ when placed on allocation $y_{i}$ and the general average weekly demand $d_{i}$. The new allocation elasticity ensures that all products will have their own allocation elasticities on different display allocations, 
instead of sharing the same fixed value with other products displayed on the same allocation.

Since facing space is limited in convenience store and most products have less than three facings on the shelves, a simplified form of the above model is proposed in order to improve the its efficiency and performance on products and product groups for convenience store. Also, due to practical difficulty of measuring the interactions between products on their display, the parameter cross-space elasticity will be ignored in the simplified model. For computational efficiency, the model will be split into two parts. The first part will assign optimal facing value to all products, and the second part will optimise the product locations only. This model formulation allows both products and product groups to be optimised simultaneously.

\section{III.NUMERICAL EXPERIMENTS}

To collect data for analysis, a three months trial is conducted in a chain store operator in Hong Kong. A total of 134 products' data are collected in this study. These 134 products will be placed on the 13 locations on the shelves. Each allocation has $121.5 \mathrm{~cm}$ length of facing space, and $1,579.5 \mathrm{~cm}$ length of facing space for whole shelves in each store. Product unit facing width, unit gross profit, average weekly demand, product group list, facing space elasticity, display area allocation elasticity are used as the input data. An optimal facing space value with products display area allocation is given with its expected total gross profit.

By changing the products' facing values and finding out the facing space elasticity on sales for different products, a matrix of average facing space elasticity of each facing value on all products can be obtained. Based on the facing space elasticity matrix, the first part of the model will be implemented to find the optimal facing value for all products. Similarly, by changing the products' allocations on the shelves and by obtaining the display area allocation elasticity on sales of all products, a matrix of average display area allocation elasticity of each display allocation on all products is given as the result. Based on the display area allocation elasticity matrix, the second part of the model will be implemented to find the optimal display area allocation for all products and product groups. By analysing the data, we observe that both facing space and display area allocation have significant effects on the sales of product.

\section{A. Optimal shelf facing space}

The first part of the optimisation will give products optimal facing value based on their facing space elasticities, and subject to the constraint of total length of shelf facing space. A series of tests are conducted to find out the best parameter settings on population size, crossover rate, mutation rate and selective strategy upon the efficiency and effectiveness when solving the optimisation problem.

The parameter setting for the best result is crossover rate equals to 0.9 (mutation rate is 0.1 ), population size equals to 300 , and use the stochastic uniform selection strategy, which gives best gross profit of HK $\$ 4,891.012$, and elapsed time of 92.285 seconds.

Compared with the result of all products without considering the shelf length constraint, the total gross profit is HK $\$ 4,915.67$, but the total width of all products' facings required is $1,651.7 \mathrm{~cm}$ (i.e. larger than the total shelf length of $1,579.5 \mathrm{~cm}$ ). By implementing the proposed approach on the constrained shelf facing space optimisation problem of store A, a $99.5 \%$ of total gross profit (acquired gross profit when there is no constraint on total shelf space length) can be obtained. Only $0.5 \mathrm{~cm}$ is over the whole shelf length constraint, which is small enough to be ignored.

\begin{tabular}{|c|c|c|c|c|c|c|c|c|c|}
\hline Selection & Stochastic & Stochastic & Stochastic & Slochastic & Stochastic & Stochostic & Slochastic & Stochastic & Stochastic \\
\hline Crossover & 0.7 & 0.8 & 0.8 & 0.7 & 0.8 & 0.8 & 0.7 & 0.8 & 0.9 \\
\hline Population & 100 & 100 & 100 & 200 & 200 & 200 & 300 & 300 & 300 \\
\hline $\begin{array}{l}\text { Elapsed } \\
\text { Time }\end{array}$ & 142.745 & 166.394 & 72.027 & 196.497 & 78.834 & 228.129 & 98.396 & 125.111 & 92285 \\
\hline Generation & 2670 & 3074 & 1558 & 2859 & 1497 & 3292 & 1579 & 1939 & 1490 \\
\hline $\begin{array}{l}\text { Gross Profit } \\
\text { (HK \$) }\end{array}$ & 4889.181 & 4890.3789 & 4885.0112 & 4881.1153 & 4881.9324 & 4886.0614 & 4885.3132 & 4891.012 & 4891.012 \\
\hline Total Width & 1580.2996 & 4579.8996 & 1578.2894 & 1578.2994 & 1579.5995 & 1578.5905 & 1580 A995 & 1580 & 1580 \\
\hline
\end{tabular}

Fig. I Performance comparison B

\section{B. Optimal display area allocation}

With the optimal shelf facing space value from
Section A, the second part of the model is to determine the optimal products display area allocations among 13 
locations on the shelves. If there is no length constraint on the display locations, which means that all products will be placed onto the area with the highest display area allocation elasticity with the optimal shelf facing space value obtained in Section 3.1, the total gross profit per week will be as high as HK\$6,447.7. The drawback of this configuration is that it will result in an unbalanced shelves layout among different display locations.

Fig. 2 gives some detailed information of the best result shelf display without length constraint on display locations. It shows that the product display among 13 different display locations. $\mathrm{L}$ and $\mathrm{R}$ is the horizontal position indicator of the allocation and the number followed indicates the vertical height of the display allocation. The number increases with the height of the allocation.

\begin{tabular}{|c|c|c|c|}
\hline Lacations & Praduct Count & Total Width (cm) & Location Length (cm) \\
\hline L1 & 12 & 102.7995 & 121.5 \\
\hline L2 & 8 & 88.1 & 121.5 \\
\hline L3 & 18 & 169.5 & 121.5 \\
\hline L4 & 8 & 89.1 & 121.5 \\
\hline L5 & 9 & 62 & 121.5 \\
\hline L6 & 21 & 187.9 & 121.5 \\
\hline R1 & 8 & 135.1 & 121.5 \\
\hline R2 & 7 & 119.7 & 121.5 \\
\hline R3 & 10 & 183.4 & 121.5 \\
\hline R4 & 9 & 113.9 & 121.5 \\
\hline R5 & 8 & 103.7 & 121.5 \\
\hline R6 & 10 & 135.2 & 121.5 \\
\hline R7 & 6 & 89.6 & 121.5 \\
\hline Sum & 134 & 1579.9995 & 1579.5 \\
\hline
\end{tabular}

Fig. 2 Product allocations without length constraint

It is obvious that some display locations attract more products compared with the other locations. However, our findings do not seem to be in perfect agreement with that of other researchers, who suggest that the eye-level shelves (between the height of knees and eyes) attract more sales compared with the other vertical shelves. The latter include (i) level 1: the lowest level at knees' height; (ii) level 3: the middle level at waist height; and (iii) level 6: the top level above eyes' height is more popular than the other locations among different products.

\begin{tabular}{||c|c|c|c|}
\hline Locations & Product Count & Total Width (cm) & Location Length (cm) \\
\hline L1 & 13 & 117.0996 & 121.5 \\
\hline L2 & 12 & 120.8 & 121.5 \\
\hline L3 & 12 & 118.2 & 121.5 \\
\hline L4 & 14 & 123.9 & 121.5 \\
\hline L5 & 13 & 120.9999 & 121.5 \\
\hline L6 & 13 & 121.7 & 121.5 \\
\hline R1 & 7 & 121 & 121.5 \\
\hline R2 & 7 & 128.7 & 121.5 \\
\hline R3 & 7 & 121.4 & 121.5 \\
\hline R4 & 9 & 123.1 & 121.5 \\
\hline R5 & 10 & 122.2 & 121.5 \\
\hline R6 & 8 & 119.7 & 121.5 \\
\hline R7 & 9 & 121.2 & 121.5 \\
\hline Sum & 134 & 1580 & 1579.5 \\
\hline
\end{tabular}

Fig. 3 Product allocations with length constraint
Fig. 3 gives some detailed information of the best result shelf display with length constraints on display locations. Although not all display locations meet their length constraint, the resulting products display allocations bring a near optimum product allocation solution, which need only some minor adjustment in or between different locations. Despite near optimum results can be obtained, the implementation of such solutions in store might not be feasible as they have not incorporated the grouping constraints. Since most stores place products in same category or sub-category together, the performance with grouping display constraints is more important for the proposed approach.

Here 1.34 products will be divided into 64 products groups, in which products need to be placed together. The comparison shows that the best result of HK $\$ 5,681.53$ is obtained with the setting of population size equals to 300 , crossover rate equals to 0.8 , and roulette-wheel is used as the selection strategy.

Compared with store's average weekly gross profit of HK $\$ 4,550$, the proposed approach could improve the weekly gross profit by almost $25 \%$. Compared with store's highest weekly gross profit of $\mathrm{HK} \$ 5,202$ in the 3 months, the proposed approach could improve the weekly gross profit by $9 \%$.

Fig. 4 shows some detailed information of the best result shelf display with length constraints and group constraints on display locations.

\begin{tabular}{|c|c|c|c|}
\hline Locations & Product Count & Total Width (cm) & Location Length (cm) \\
\hline L1 & 13 & 119.2996 & 121.5 \\
\hline L2 & 16 & 127.9 & 121.5 \\
\hline L3 & 12 & 121.5 & 121.5 \\
\hline L4 & 13 & 118.4 & 121.5 \\
\hline L5 & 14 & 122.3999 & 121.5 \\
\hline L6 & 9 & 117.6 & 121.5 \\
\hline R1 & 8 & 131.9 & 121.5 \\
\hline R2 & 7 & 115 & 121.5 \\
\hline R3 & 7 & 114.8 & 121.5 \\
\hline R4 & 7 & 120.7 & 121.5 \\
\hline R5 & 12 & 124 & 121.5 \\
\hline R6 & 8 & 124.9 & 121.5 \\
\hline R7 & 8 & 121.6 & 121.5 \\
\hline Sum & 134 & 1580 & 1579.5 \\
\hline
\end{tabular}

Fig. 4 Product allocations with grouping constraint

Because of the grouping constraints, products need to be placed by their groups, some products may need to be reallocated based on their size, to make the whole shelf looks balanced and comfortable.

The analysis shows that the proposed approach could improve the current average weekly gross profit by $20 \%$. Although the optimal result given by the proposed approach may still need some modifications between different display locations before its implementation on store shelves, it enables people to have a general guideline before they plan the shelf layout, which will save a lot of time in implementing planograms. 


\section{CONCLUSION}

The model developed in this study emphasises both the importance of facing space and display area allocation effects on sales, and of the minimal requirement of human intervention (on the estimation of a vast number of parameters) for easy implementation. Unlike recent models that just use small size hypothesis data sets in their verification, the proposed model is implemented and tested rigorously using a relatively large amount of store data, which is acquired from a 3 months store trial. A total 134 drink and food products from 30 categories under 3 departments and $3,159 \mathrm{~cm}$ length of shelf space from a store operator is involved in the study. This store trial design can be easily implemented in different stores; retailers could take it as a road map for the analysis of facing space and display area allocation. Also, an integer encoding GA program is also developed to tackle the store shelf space optimisation problem.

\section{REFERENCES}

[1] Desmet, P. and V. Renaudin (1998). "Estimation of product category sales responsiveness to allocated shelf space." International Journal of Research in Marketing 15(5): 443-457.

[2] Dreze, X., S. J. Hoch, et al. (1994). "Shelf management and space elasticity." Journal of Retailing 70(4): 301-326. 